\title{
Extreme ultraviolet (EUV) solar spectral irradiance (SSI) for ionospheric application - history and contemporary state-of-art
}

\author{
G. Schmidtke ${ }^{1}$, Ch. Jacobi ${ }^{2}$, B. Nikutowski ${ }^{1,2}$, and Ch. Erhardt ${ }^{1}$ \\ ${ }^{1}$ Fraunhofer Institute for Physical Measurement Techniques IPM, Heidenhofstrasse 8, 79110 Freiburg, Germany \\ ${ }^{2}$ Institute for Meteorology, University of Leipzig, Stephanstr. 3, 04103 Leipzig, Germany \\ Correspondence to: G. Schmidtke (gerhard.schmidtke@ipm.fraunhofer.de)
}

Received: 18 December 2013 - Revised: 27 February 2014 - Accepted: 14 March 2014 - Published: 11 November 2014

\begin{abstract}
After a historical survey of space related EUV measurements in Germany and the role of Karl Rawer in pursuing this work, we describe present developments in EUV spectroscopy and provide a brief outlook on future activities. The group of Karl Rawer has performed the first scientific space project in Western Europe on 19th October 1954. Then it was decided to include the field of solar EUV spectroscopy in ionospheric investigations. Starting in 1957 an intensified development of instrumentation was going on to explore solar EUV radiation, atmospheric airglow and auroral emissions until the institute had to stop space activities in the early nineteen-eighties. EUV spectroscopy was continued outside of the institute during eight years. This area of work was supported again by the institute developing the Auto-Calibrating Spectrometers (SolACES) for a mission on the International Space Station (ISS). After more than six years in space the instrument is still in operation. Meanwhile the work on the primary task also to validate EUV data available from other space missions has made good progress. The first results of validating those data and combine them into one set of EUV solar spectral irradiance are very promising. It will be recommended for using it by the science and application community. Moreover, a new low-cost type of an EUV spectrometer is presented for monitoring the solar EUV radiation. It shall be further developed for providing EUV-TEC data to be applied in ionospheric models replacing the Covington index $F_{10.7}$. Applying these data for example in the GNSS signal evaluation a more accurate determination of GNSS receiver positions is expected for correcting the propagation delays of navigation signals traveling through the ionosphere from space to earth. - Latest results in the field of solar EUV spectroscopy are discussed, too.
\end{abstract}

\section{Introduction}

During his activities in the Ionosphären-Institut Breisach, in 1957 Karl Rawer decided to start research in the field of solar radiation that is most strongly controlling the ionosphere flowed by the interaction with solar wind phenomena. To those times the knowledge about the dependence of the electron densities on the solar activity was well established, but the relation between variability of solar extreme ultraviolet (EUV) radiation and the ionospheric response was not at all known, neither qualitatively nor quantitatively. To learn more about the varying solar EUV energy continuously deposited in the ionosphere was the task of a new group working in the area of the University of Freiburg.

\section{Historical survey}

After the successful measurement by a radio spectrometer aboard a French Veronique rocket on 19th October 1954 - it was the first scientific rocket mission of Western Europe - Rawers team added an Impedance Probe and a Seddon Experiment (measuring the phase delay time) to the next rocket payload in 1957 supporting ionospheric sounding from ground.

The student Frank Ade started EUV spectroscopy for ionospheric application in line with a diploma thesis in 1957. It took three years to record spectra in the vacuum ultraviolet and in the extreme ultraviolet region. After Ade received the diploma degree he left the laboratory. The students Gerhard Schmidtke, Fritz Fischer and Hans-Joachim Boehnel continued the work. However, lacking of experience in EUV spectroscopy it was quite difficult to start working on the final 


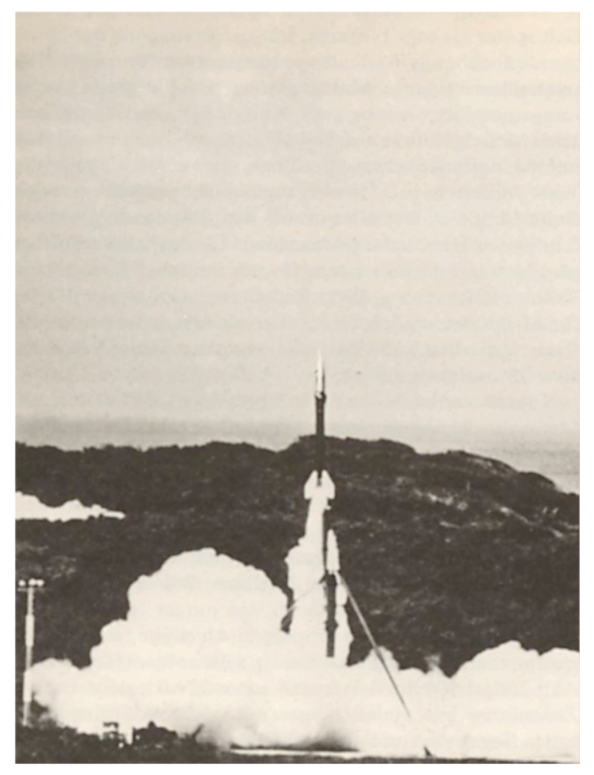

Figure 1. Launch of a Veronique rocket from Kourou/Guayana.

goal that was the development of EUV space instrumentation.

Moving from the university district to an old villa Karl Rawer established the Arbeitsgruppe für Physikalische Weltraumforschung (APW) in 1963. The laboratory vacuum spectrometer, a shaker for load qualification of instruments, some mechanical and electronic equipment such as a oscilloscope supported the work for the realization of first ideas bringing together EUV and plasma instrumentation to in-situ investigating the ionosphere.

In 1963/1964 Schmidtke received a fellowship to join the U.S. Air Force group lead by Hans Hinteregger in Cambridge Massachussetts/USA. Hinteregger was a pioneer developing space-born EUV instruments based on counting spectrogram technique. After launching several Aerobee rockets the results of the science data evaluation were summarized and published in a tabulation of solar EUV fluxes of the spectral range from $177.5 \mathrm{~nm}$ to $1 \mathrm{~nm}$ (Hinteregger et al., 1965). In the thermospheric-ionospheric research community this data set was used as EUV standard flux for years. A close cooperation between the two groups continued for many years.

The generous support by Hinteregger for a successful work of the young and ambitious APW resulted in the straight-forward development of a rocket EUV spectrometer. Even more, a sensor of a retarding potential analyzer crossed the Atlantic Ocean for further developing and using it in a combined payload measuring the EUV energy influx and plasma parameters such as electron density and temperature and supra-thermal electron fluxes as well as ion density and ion distribution in the thermospheric-ionospheric system.

Since 1st October 1964 the APW was administrated by the Fraunhofer Gesellschaft für Angewandte Forschung. Based

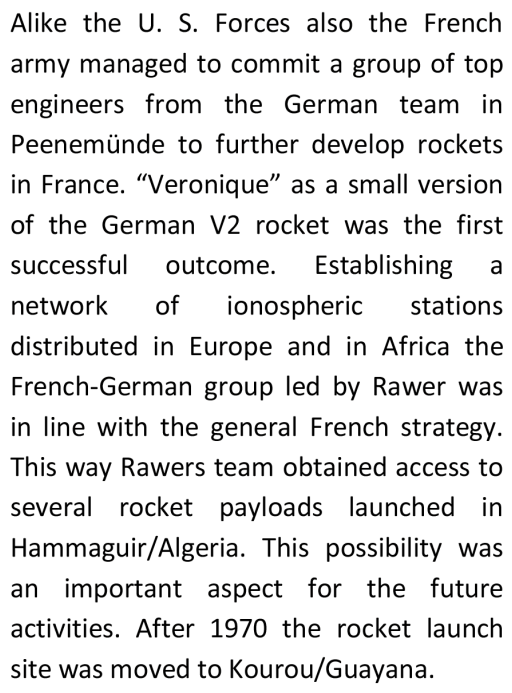

on the grant WRK 50 (Weltraumkunde) from the Ministry of Education and Science (BMBW) up to 20 young enthusiasts of space research started designing, developing, and fabricating instruments for rocket payloads. After several rocket missions with the Radiospectrometer, the Seddon Experiment and the Impedance Probe an EUV counter tube was the first experiment to detect solar radiation (Fischer, 1968). The rocket missions served also for qualifying and verifying of instruments to be operated aboard satellites in future projects.

In the laboratory good progress was made in the field of EUV spectroscopy related to space research. With the financial support by the BMBW versatile equipment facilitated the space activities (Schmidtke, 1968). A big vacuum chamber was equipped with a new type of differential pumping system (Schmidtke, 1971) and with different light sources simulating the solar radiation from the X-ray through the UV spectral regions. The $\mathrm{X}$-ray device generated characteristic emissions and bremsstrahlung from five anti-cathodes of different materials. The development of EUV hollow cathode discharges using materials such as wolfram, tantalum, and water-cooled aluminum provided a broad selection of spectral emissions from different gases or gas mixtures. For both types of laboratory instruments a power convertor supplied the radiation sources with up to $1.5 \mathrm{~kW}$ at voltage levels from $15 \mathrm{kV}$ down to some hundred volts. Also the detectors available covered the full spectral range of interest. Counting technique with secondary electron multipliers as well as with channel electron multipliers and analog current recording represented the state of the art of those days.

After testing and qualifying the experiment an EUV monochromator recorded the altitude profile of a 


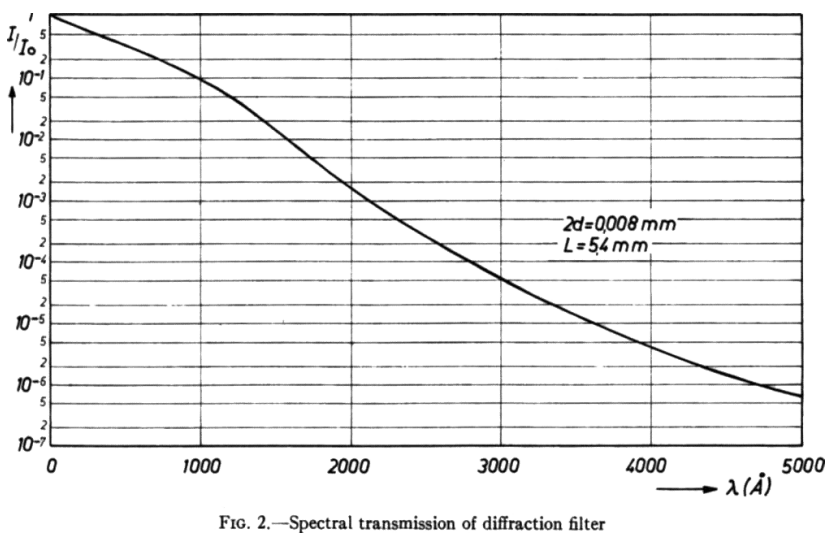

Figure 2. Typical transmission characteristic of a diffraction filter ( $x$ axis in Angstrøm units) (Schweizer and Schmidtke, 1971).

molecular nitrogen emission in a polar aurora event over Kiruna/Sweden in 1970 (Landensperger, 1971).

The new facilities allowed testing and calibrating of sophisticated components and instruments. The development of the diffraction filter technology (see Fig. 2) made good progress (Schmidtke, 1968; Schmidtke, 1968/69; Schmidtke, 1970). In cooperation with Loren Actons group at Lockheed, Palo Alto, USA, a rocket payload for spectral recording of emissions has been developed. During the rocket mission of the high-resolution XUV diffraction filter spectrograph (Bruner et al., 1979; Schweizer and Schmidtke, 1971) approximately 50 solar emission lines of the EUV spectrum between $5 \mathrm{~nm}$ to $21.2 \mathrm{~nm}$ have been recorded on a Kodak SC-7 film with a spectral resolution of about $0.05 \AA$ (see Figs. 3 and 4).

With the rocket launches from Hammaguir/Algeria, Sardinia/Italy, Andoya/Norway, Kiruna/Sweden, Wallops Island/USA, White Sands/USA and other places and with the presentation of the scientific results on international conferences such as COSPAR, URSI, SCOSTEP, IAGA, AGU, EGU and others the APW gained reputation in the space science community.

1968 the BMBW negotiated with NASA for working on a German-American satellite mission exploring the ionospheric-thermospheric system. Given the international cooperation of the APW as well as the possible contribution of his group a proposal for building and launching an aeronomy satellite called AEROS was elaborated. Five active experiments adding to each other were selected,

- Neutral Mass Spectrometer of Goddard Space Flight Center in Greenbelt, MD, USA,

- Ion Mass Spectrometer of the Max-Planck-Institut in Heidelberg, Germany,

- Retarding Potential Analyzer of APW in Freiburg, Germany,

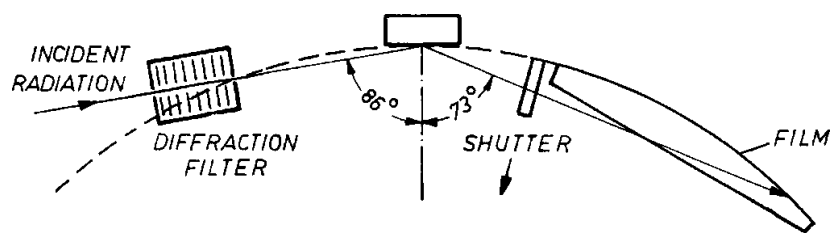

Figure 3. The diffraction filter acted as the entrance slit of the grazing incidence spectrograph (Schweizer and Schmidtke, 1971).
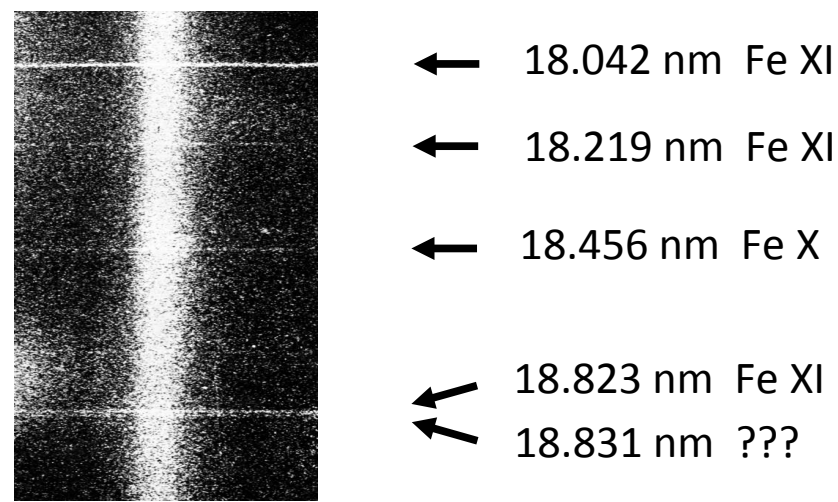

Figure 4. Selected interval of the solar spectrum recorded with the grazing incidence spectrograph on 29th April 1971 (after Schweizer and Schmidtke, 1971).

- Impedance Probe of APW in Freiburg, Germany, and

- EUV spectrometer of APW in Freiburg, Germany,

plus the passive experiment Satellite Drag of Astronomische Institute of university Bonn, Germany.

These activities required the recruitment of the personnel. However, there was too little space in the rented accommodation of three old residential houses for the 30 employees. Therefore, 1971 the kick-off for constructing a new building envisaged the release of these limitations. In the plan the new building provided space for 49 members of staff and ample space for laboratory equipment with a large plasma simulation chamber and a large auroral/airglow simulation chamber included. Working on the Veronique payloads, on the three instruments of the AEROS satellite, on developing and building the instrumentation of three rocket missions for adding altitude profiles of aeronomic parameters to the horizontally measuring AEROS instruments, on other rocket instrumentation and on the procuration of the facilities for the new laboratories - all these activities required the full engagement of the colleagues carrying out many hours of overtime.

1971 both Veronique missions acquired good results. At the same time the Veronique instruments received the official verification label for the AEROS satellite mission. End of 1972 the satellite was launched successfully.

With the operation of the satellite AEROS new philosophies of project attendance commenced requiring teams being all times available. Another task challenged the scientists. 


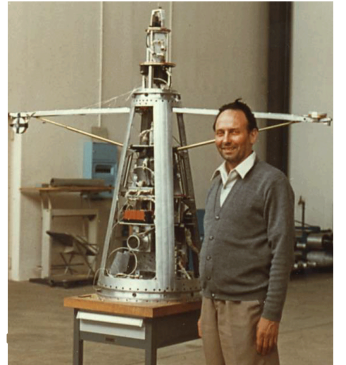

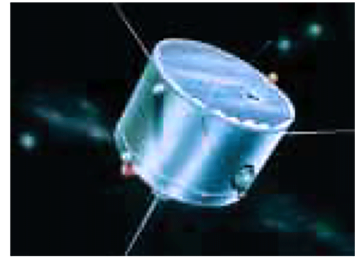

Dornier Company in Friedrichshafen constructed, tested and qualified the AEROS satellite. Support was also given by Dornier during the launch preparation and during the launch of AEROS on $16^{\text {th }}$ December 1972 in Vandenberg/California. The mission lasted till 22nd August 1973.

Figure 6. Picture of the AEROS satellite.

the large photocathodes of the Bendix multipliers were exposed to a nickel 63 radioactive beta source (Schmidtke et al., 1974).

During the solar minimum that occurred in 1975 an unexpected change of the solar EUV flux was observed with a distinct minimum. During this period a rather poor correlation between the EUV fluxes and the $F_{10.7} \mathrm{~cm}$ index was noted.

Based on the AEROS findings and on those from other missions it was decided to start the follower mission of AEROS-B with the mission period lasting from July 1974 to September 1975.

End of 1979 Karl Rawer retired. In his institute a great number of experiments with the data evaluation have been accomplished: 15 different scientific instruments provided the basis for 121 rocket and 4 satellite experiments. In addition the Retarding Potential Analyzer measured interplanetary and ionospheric plasma parameters aboard the Pioneer Venus Orbiter.

In 1980 the institute was renamed to Fraunhofer Institut für Physikalische Messtechnik (IPM) with a change of the fields of research. Space research was outsourced and stopped in the institute. The still on-going satellite project Airglow-Solar Spectrometer Instrument (ASSI) to be flown aboard the Italian-American San Marco 5 satellite has been worked on with Physikalisch-Technische Studien GmbH in the Karl-Rawer-Haus (Schmidtke et al., 1985). The Principal Investigator had the chance to lead the experiment outside of the IPM. In close cooperation with Tom Woods of Boulder an EUV-UV reference spectrum from $20 \mathrm{~nm}$ to $200 \mathrm{~nm}$ has been elaborated (Schmidtke et al., 1992).

Until the end of the 20th century no data set has been available covering the spectral EUV range of aeronomic interest from at least $10 \mathrm{~nm}$ to $105 \mathrm{~nm}$ during a full solar cycle. Even the data recordings available did not produce reliable data with respect to radiometric accuracy due to many sources of degradation in the instruments as well as from the environment of spacecraft. EUV spectrophotometry is a field of surface effects. Among many other effects from the space environment the EUV photons with energies exceeding $80 \mathrm{eV}$ themselves do change the surfaces such that it is not possible to determine the efficiency parameters of the optical components like optical gratings, mirrors and detectors. Hence, there was no way to separate solar activity from instrumental efficiency changes at a level achieving an absolute accuracy 


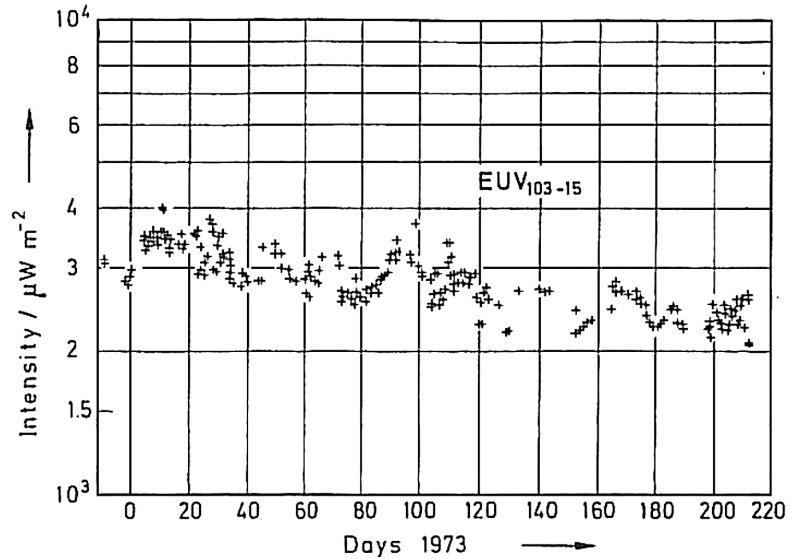

EUV indices have been proposed representing the EUV irradiance from the whole solar disk at Earth's distance (Schmidtke, 1976) in units of $W \mathrm{~m}^{-2}$. For single line emissions the denotation is $\operatorname{EUV}_{X}$ with $X$ wavelength. For intervals $E_{U} U V_{Y-Z}$ is defining the irradiance of the wavelength interval $Y$-Z.

Figure 7. Solar flux index EUV $103-15$, i.e. the flux intensity in the main EUV range of ionospheric interest (Schmidtke, 1984).
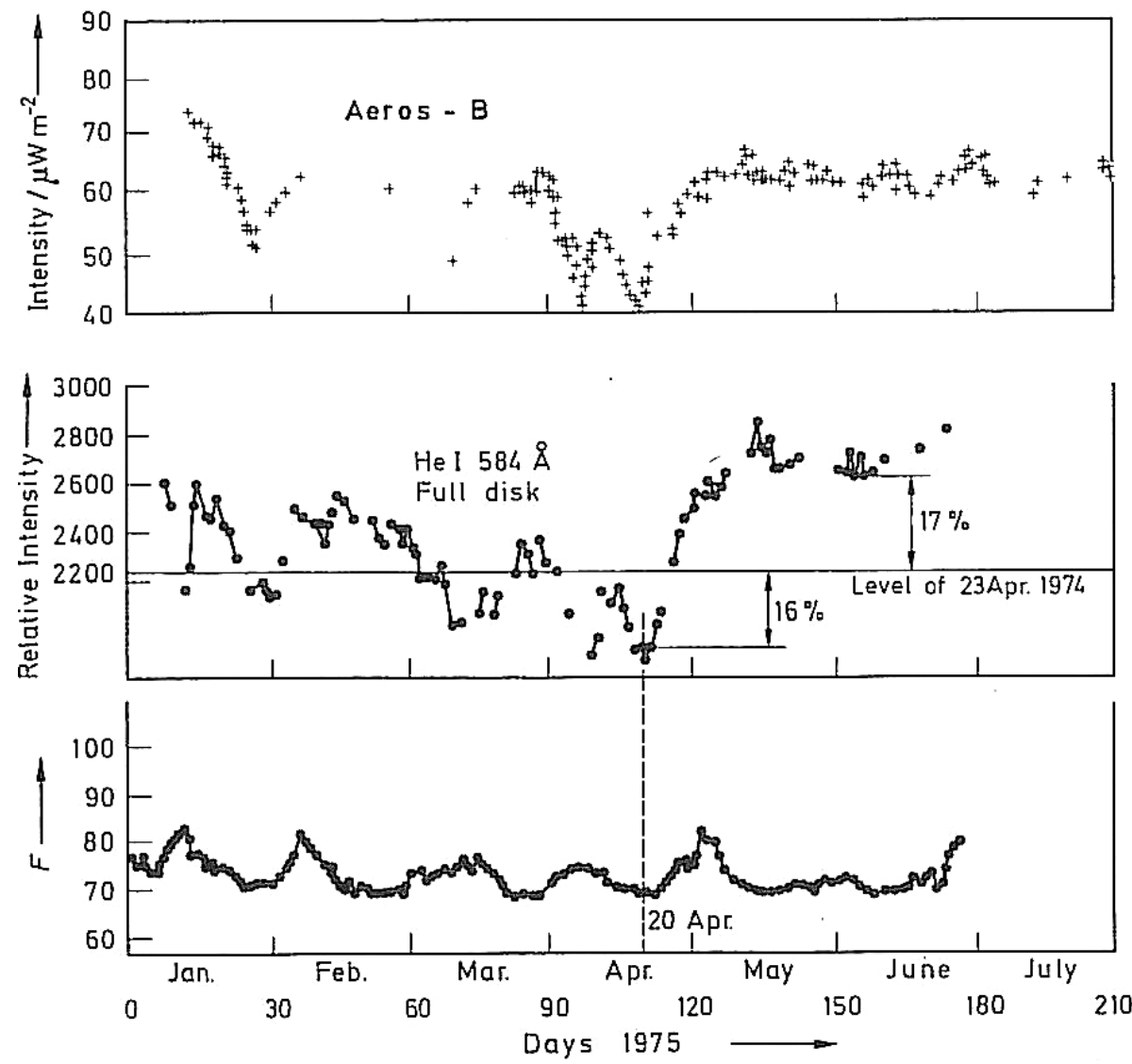

Figure 8. The solar "EUV Minimum" between solar cycles 20 and 21 in April 1975 observed at 58.4 nm (He I) on satellites AE-C and AEROS-B (Schmidtke, 1984).

of about $10 \%$ or even less. For example, modeling the data from the satellite SNOE (Solomon et al., 2000) solar flux data as published by Hinteregger et al. (1981) had to be multiplied by a factor of four below $25 \mathrm{~nm}$ for matching SNOE observations and modeling.
In summary, it was not possible to provide a set EUV data as required for thermospheric-ionospheric modeling covering a full solar cycle and ensuring a radiometric accuracy of the order of $10 \%$. Also the availability on time could not yet be realized due to the complex data evaluation. 

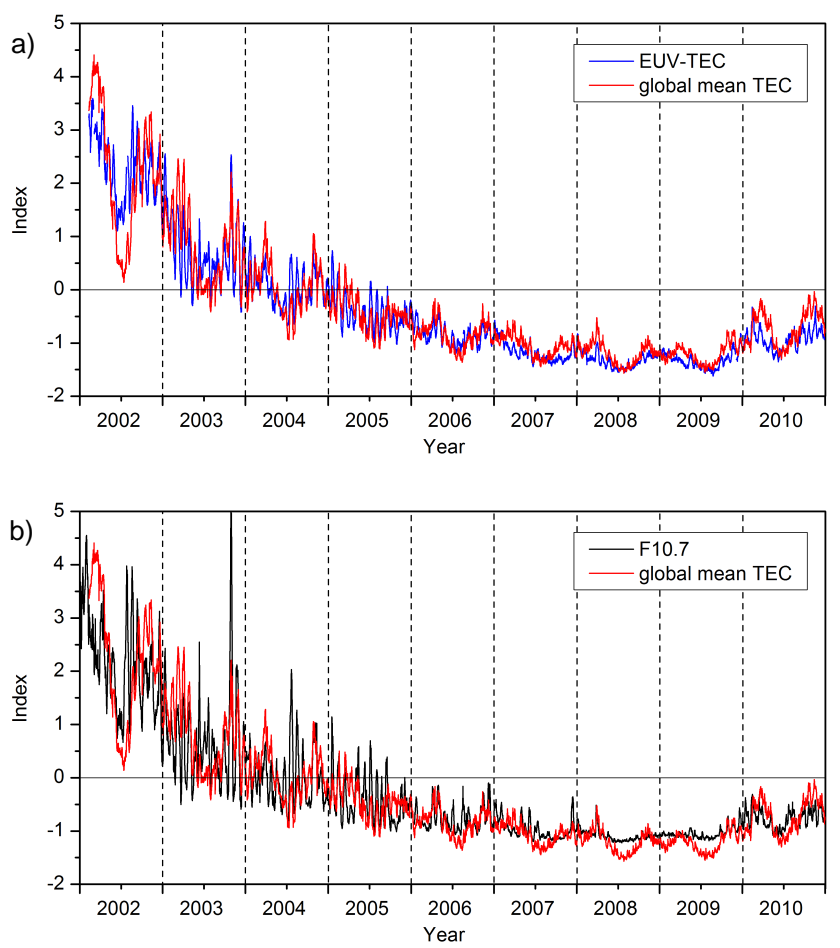

Figure 9. Time series of computed daily global TEC values based on measured daily EUV-TEC indices are compared with measured global mean TEC data in the upper panel. Time series of computed daily global TEC values based on the $F_{10.7}$ index are compared with measured global mean TEC data in the lower panel. Data are normalised by mean and standard deviation from 2002-2007 (after Unglaub et al., 2011).

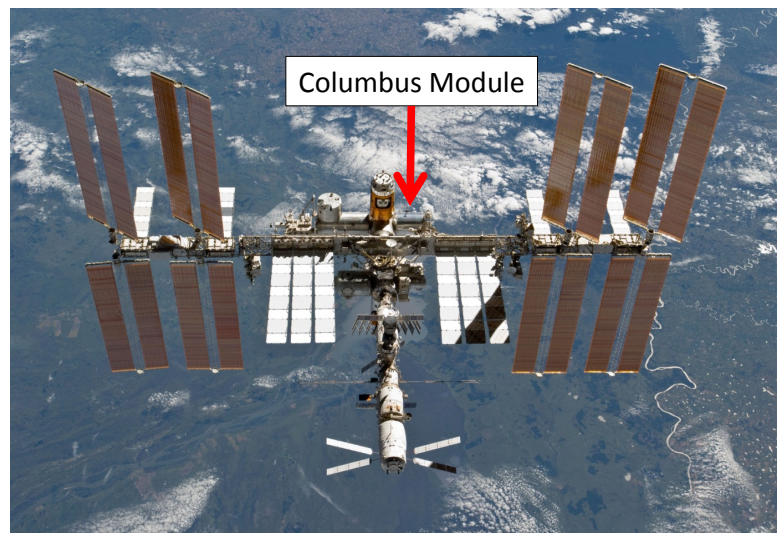

Figure 10a. The International Space Station and the Columbus Module (Courtesy of NASA/ESA).

\section{Solar EUV spectroscopy: state of the art}

With the launch of the satellite TIMED in December 2001 (Woods et al., 2005), of the payload ISS-SOLAR aboard the Columbus Module at the International Space Station (ISS) with the instrument SolACES in April 2008 (Schmidtke et

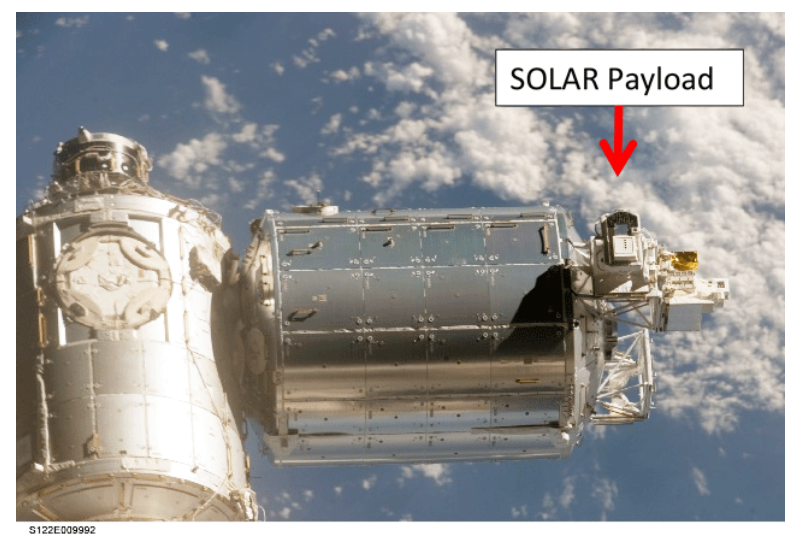

Figure 10b. The SOLAR payload at the Columbus Module (Courtesy of NASA/ESA).
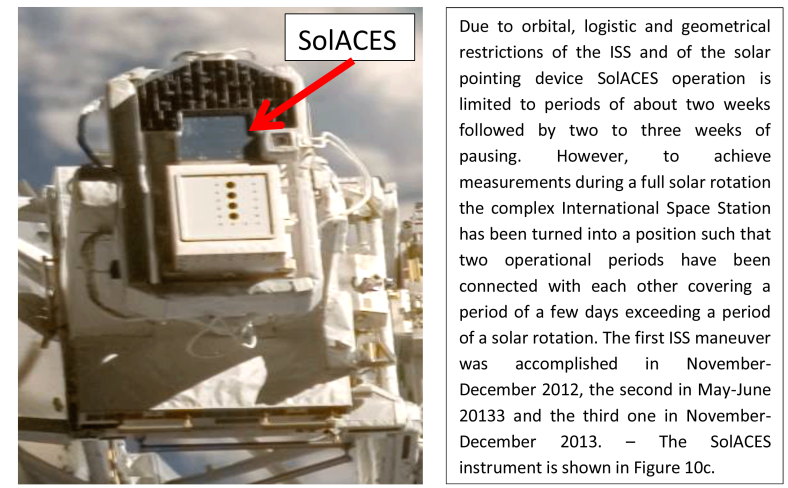

Figure 10c. The ISS/SolACES instrument at the SOLAR payload (Courtesy of NASA/ESA).

al., 2014), and of the satellite SDO with the instrument EVE in May 2010 (Woods et al., 2012) a new approach re-calibrating the EUV spectrometers during the mission started. While TIMED/SEE and SDO/EVE instruments are cross-calibrated once per year against a rocket spectrometer calibrated at a synchrotron primary radiation standard on ground, SolACES is demonstrating the in-orbit re-calibration capability of EUV spectrometers in space. The latter instrument is applying ionization chambers as primary detector standard. This way re-calibration can be achieved every day, if required. The EUV data as recorded by these three and other instruments since the implementation of TIMED/SEE allow to assemble a full set EUV data covering one period of a solar cycle. Based on the improved calibration procedures applied the new data allow to make more progress in upper atmospheric physics and applications such as spacecraft and debris orbit prediction and ionospheric modeling. In Fig. 9 the global normalised mean photoionisation rate data (which should be broadly proportional to TEC) based on actual measurements are compared with the computed global mean TEC based on EUV measurements (upper graph) and with the computed global mean TEC based on the $F_{10.7}$ index. 


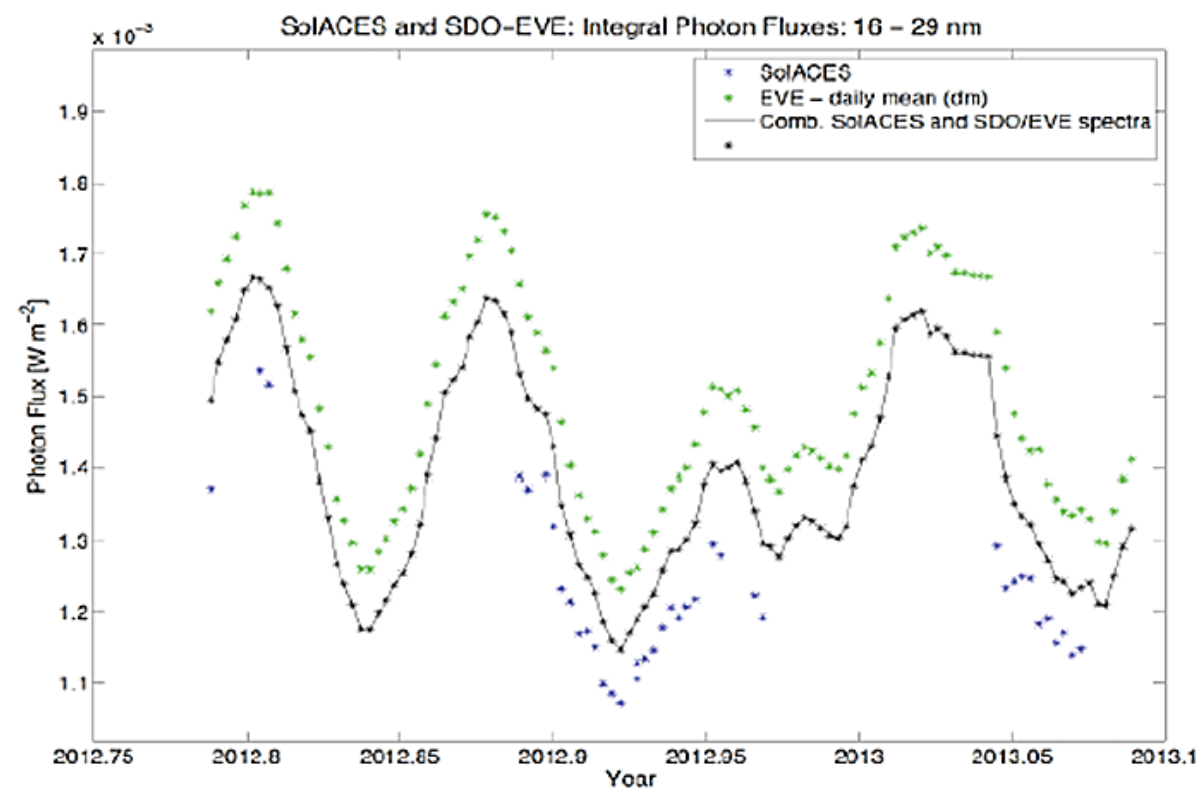

Figure 11a. SDO/EVE flux data (green dots) and ISS/SolACES flux data (blue dots) are averaged (black dots) for nearly four solar rotations in November 2012-January 2013.

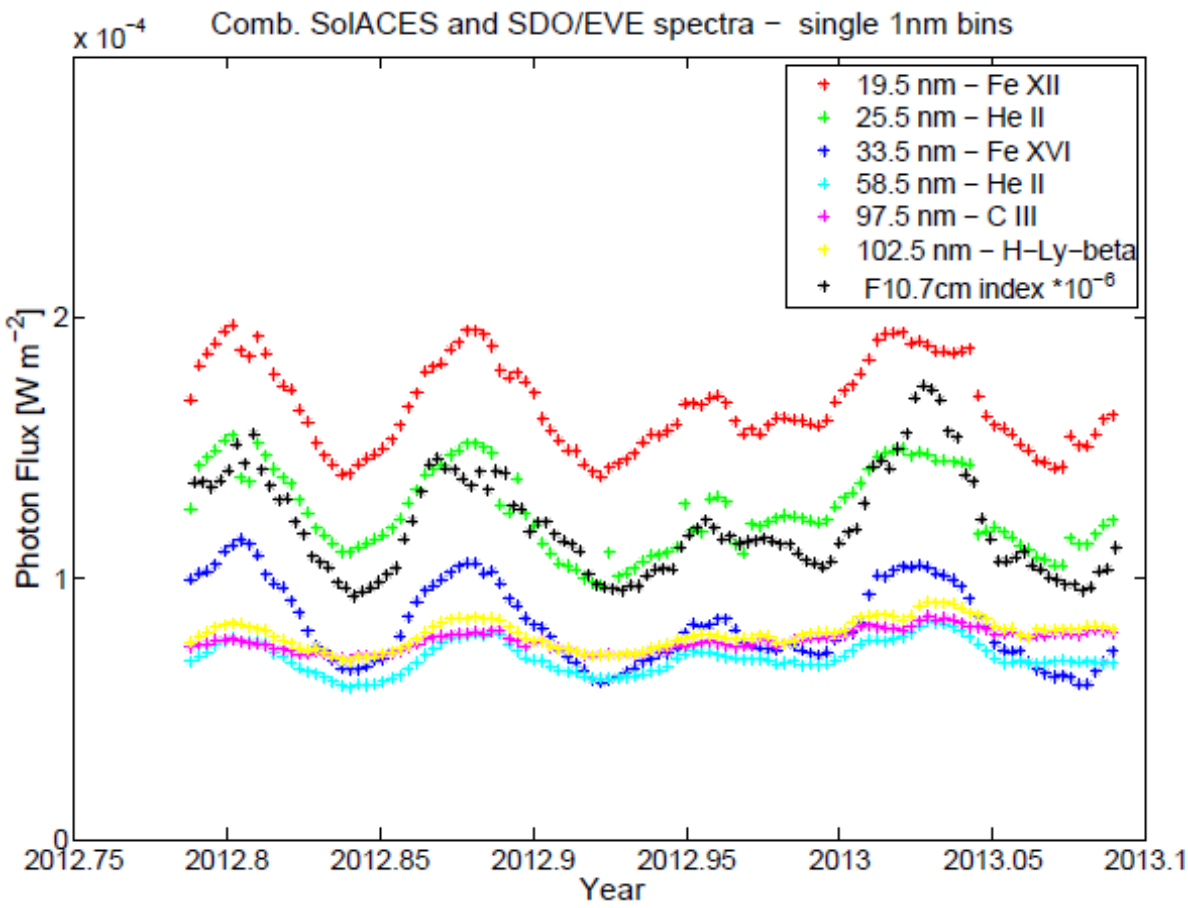

Figure 11b. Selected wavelengths of the combined SolACES and SDO/EVE flux data are compared with the $F_{10.7} \mathrm{~cm}$ index (see + marking in black color) for nearly four solar rotations in November 2012-January 2013.

The correlation of the measured with the computed TEC values is higher with the EUV data than with the $F_{10.7}$ index. Deviations are most pronounced during solar minimum activity (Unglaub et al., 2011). The contribution of solar parti- cle fluxes to the global TEC values is not taken into account in the computed TEC data.

In view of the applications of spectral solar EUV irradiance data there is grown interest in two aspects. In order to apply EUV data in ionospheric and thermospheric modeling 


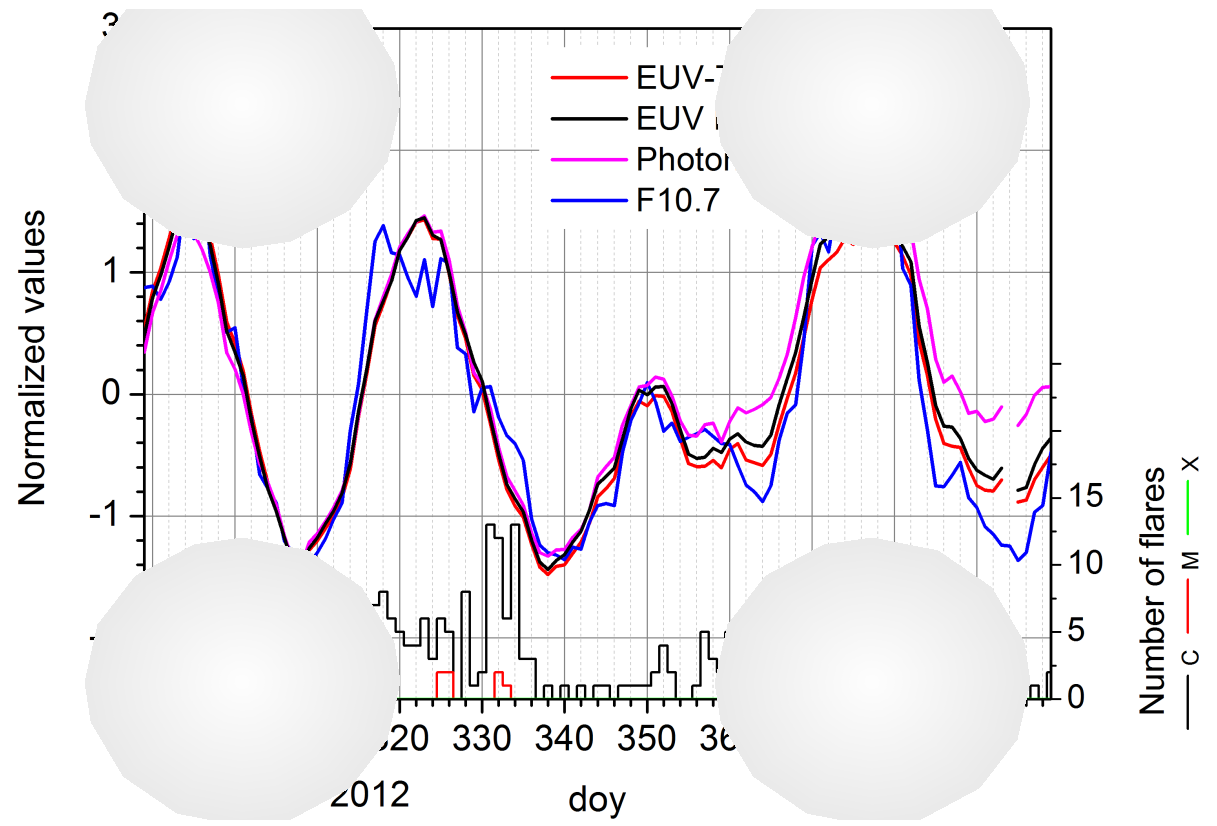

Figure 12. EUV-TEC proxy (red), based on combined SolACES and SDO-EVE spectra (17-105 nm) as shown in Fig. 11. Also shown are EUV flux (black) and photon fluxes (magenta). The data are based on EUV spectra from $17-105 \mathrm{~nm} . F_{10.7}$ (blue) solar proxy is also added. All values are normalised by the data from day \#299 through day \#326 (approx. Carrington rotation \#2143). In the lower part, the daily number of flares is shown.

and in related fields higher radiometric accuracy of longterm data sets is required. With the advent of world-wide use of satellite-born navigation systems the implementation of EUV-based ionospheric models for correcting the temporal retardation of the signals on its way from the GNSS satellite to the detectors on ground required is calling for on-line measurements of the solar EUV fluxes. In view of these requirements the following part is focused on the data derived from the three instruments mentioned before.

The instruments TIMED/SEE and SDO/EVE provide EUV data almost for every day. SEE is operating since beginning of 2002 and EVE started measurements in May 2010. ISS-SOLAR/SolACES is launched in April 2008 measuring periodically with regular ISS-SOLAR operational gaps between the solar viewing windows (see below). These three instruments are still in operation.

Though the spectrometers are cross-calibrated (SEE and EVE once per years) and re-calibrated (SolACES up to every day during operation), degradation of the instruments is still a problem.

In order to elaborate a reference data set to propose for use in the science and application communities SDO/EVE and ISS-SOLAR/SolACES solar flux data of the spectral interval from $16 \mathrm{~nm}$ to $29 \mathrm{~nm}$ and of some selected solar emissions are averaged as a first approach (see Figs. 11a and b). The range of uncertainty of both data sets is quoted to about $10 \%$.

In Fig. 12, the number of photons, EUV flux, and calculated ionization (EUV-TEC) is shown together with the $F_{10.7}$ index. These data are normalized by the values from one solar rotation. In general, $F_{10.7}$ fairly well reproduces EUV variability. During times of enhanced solar flare activity, however, $F_{10.7}$ tends to overestimate the EUV while during times of low solar activity $F_{10.7}$ tends to underestimate the EUV variability (see Fig. 9). The data set will be extended up to the latest measurements and back to the first measurements accomplished by TIMED/SEE. This way it is expected to compile an EUV data set covering more than 11 years.

\section{EUV spectroscopy: future application in GNSS data evaluation}

With the presentation of an EUV data set covering 11 years studies of implementation EUV data in ionosphericthermospheric and semi-empirical EUV modeling can be started with the goal to provide improved EUV fluxes for a wide range of applications. Because providing measured EUV SSI data is still a complex and costly task, it is indispensable to develop a reliable low-cost EUV detector providing EUV data on-line. For GNSS data evaluation such a system should be placed aboard the GNSS satellites. It shall provide EUV data directly for computation of ionospheric TEC values and feeding the navigation data with a correction for the propagation delays of navigation signals traveling through the ionosphere from space to earth.

Instead of deriving EUV data from sophisticated EUV instruments with optical surfaces sensitive to degradation the 


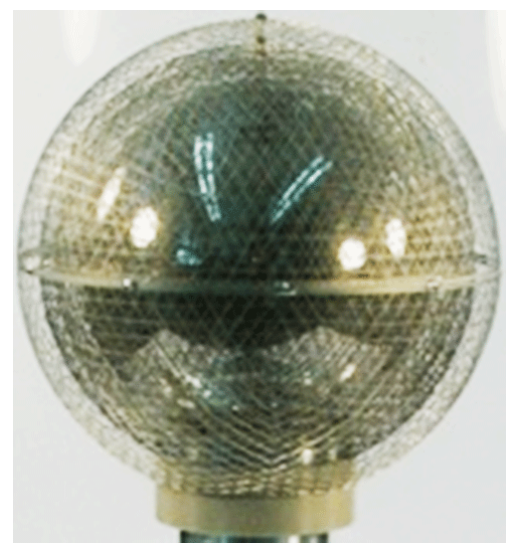

Figure 13a. Laboratory sample of a SEPS sensor.

Spherical EUV and Plasma Sensor (SEPS) is providing one possibility for EUV monitoring. The device consists of three isolated spheres. A highly transparent Inner Grid (IG) and an Outer Grid (OG) are placed around the metallic sphere (MS) with a long-term low level of degradation forming three electrodes (Fig. 13). Each one is being connected to sensitive floating electrometers. EUV photons hitting the metallic sphere generate photoelectrons. Varying the voltage potential of the metallic sphere while the potentials of the outer and inner grids are constant, the evaluation of the photoelectron currents from the three electrodes allow to derive the incoming EUV photon flux. This sensor has been tested in the ESTEC plasma chamber in combination with a Langmuir Probe, in the SolACES test chamber and at the BESSY electron synchrotron.

The development of a low-cost EUV sensor is one of the requests of the TIGER program (Schmidtke et al., 2006). The combination with sophisticated instrumentation like SEE, EVE, SolACES or Solar Patrol (Avakyan, 2006) would meet the most important requirements for the next decade.

\section{Summary}

After 50 years of SSI EUV research there is a promising work going on to provide the first validated set of EUV data covering more than 11 years comparable with the period of a solar cycle. This data set is being established with SOHO/SEM, TIMED/SEE, SDO/EVE, and ISS-SOLAR/SolACES data. It shall be published at the TIGER/COSPAR Symposium in Moscow 2014.

From the first set of data covering three solar rotations as shown in Fig. 11 an EUV TEC proxy has been derived (Fig. 12) as described by Unglaub et al. (2011). It will be further tested and correlated with ionospheric data from ground based measurements at the International Centre for Theoretical Physics in Trieste, Italy.

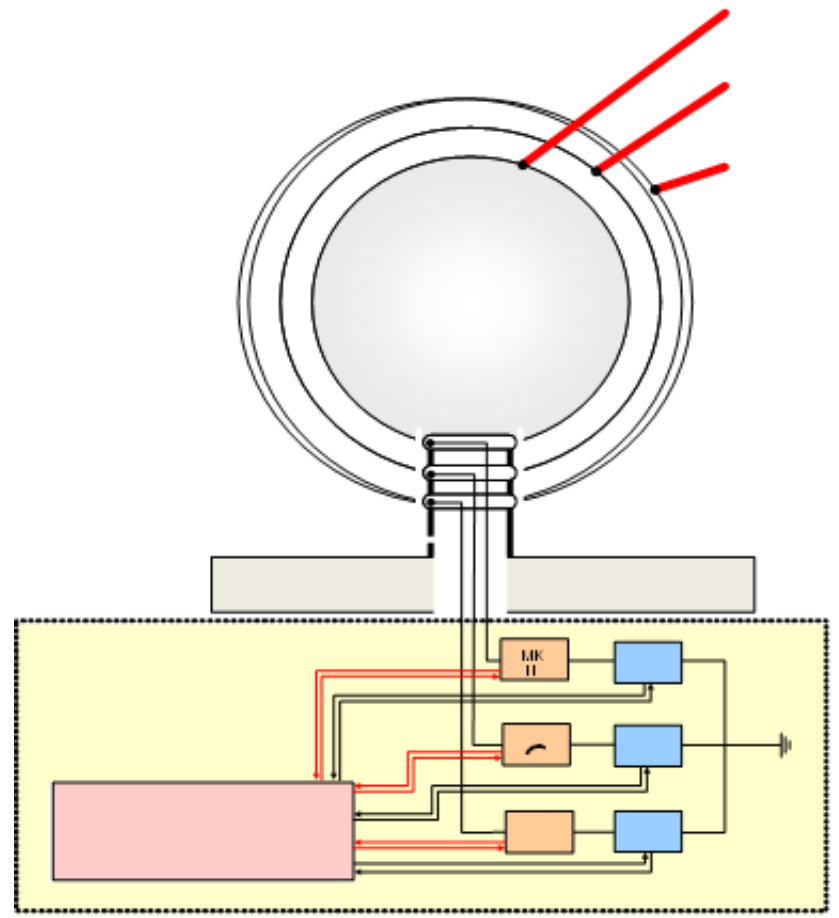

Figure 13b. Schematics of the SEPS Sensor - three blue areas: voltage Supplies; three orange areas: ampere meters; pink area: control electronics and data management.

As next step the on-orbit verification of the SEPS sensor for producing online EUV data is envisaged for later use aboard GNSS satellites for direct signal processing replacing $F_{10.7}$ by an EUV-TEC proxy.

Acknowledgements. Gerhard Schmidtke wishes to acknowledge the unbroken support by Professor Dr. Dr. h. c. Karl Rawer from 1960 until his retirement end of 1979. Until these days it has been and it still is always a great pleasure to meet and to communicate with this open-minded and well-known scientist.

The SolACES project is sponsored by DLR Bonn, by ESA/ESTEC Noordwijk and by the Fraunhofer Institute for Measuring Technique.

We thank C. Unglaub and J. Hein, Leipzig, for preparing EUV-TEC data. Flare numbers have been provided by NOAA, Space Weather Prediction Center, through http://www.swpc.noaa. gov/ftpdir/indices/. $F_{10.7}$ solar indices have been provided by NGDC through ftp://ftp.ngdc.noaa.gov/STP/SOLAR_DATA/.

We greatly appreciate the stimulation provided by the referees Prof. Andrei Mikhailov and Prof. Jens Taubenheim improving the content of this publication.

Edited by: M. Förster

Reviewed by: A. Mikhailov and J. Taubenheim 


\section{References}

Avakyan, S. V.: Solar Patrol: Absolute measurements of ionizing solar radiation, Adv. Space Res., 37, 297-302, 2006.

Bruner Jr., E. C., Acton, L. W., Brown, W. A., Salat, S. W., Franks, A., Schmidtke, G., Schweizer, W., and Speer, R. J.: X-ray spectrometer spectrograph telescope system, Proceed. Society PhotoOptical Instrumentation Engineers, 184, 270-277, 1979.

Fischer, F.: Erfahrungen mit offenen UV-Zählrohren im Wellenlängenbereich zwischen 900 und $1300 \AA$ A. Optik, 27, 1-4, 1968.

Hinteregger, H. E., Hall, L. A., and Schmidtke, G.: Solar XUV radiation and neutral particle distribution in July 1963 thermosphere, Space Res., V, 1175-1190, 1965.

Hinteregger, H. E., Fukui, K., and Gilson, B. R.: Observational reference and model data on solar EUV from measurements on AEE, Geophys. Res. Lett., 8, 1147-1150, 1981.

Landensperger, W.: Auroral luminosity profile in the far ultraviolet, Space Res., XI, 1190-1195, 1971.

Schmidtke, G.: Soller-Blendensysteme als Kollimatoren und Beugungsfilter, Zeitschrift für angewandte Phys., 25, 314-318, 1968.

Schmidtke, G.: Spektrale Anpassung von Beugungsfiltern durch den Strahlungsbündelungseffekt, Optik, 28, 578-584, 1968/69.

Schmidtke, G.: EUV-Laborspektrophotometrie zur Vorbereitung von optischen Flugexperimenten, Zeitschrift für Geophysik, 35, 69-82, 1969.

Schmidtke, G.: Diffraction filters in XUV spectroscopy, Appl. Phys., 9, 447-450, 1970.

Schmidtke, G.: New techniques for differential pumping systems, The Rev. of Scient. Instrum., 42, 1431-1433, 1971.

Schmidtke, G.: EUV indices for solar-terrestrial relations, Geophys. Res. Lett., 3, 573-576, 1976.

Schmidtke, G.: Modeling of the solar extreme ultraviolet irradiance for aeronomic applications, Encyclopedia of Physics, XLIX/7: Geophysics III, Part VII, edited by: Rawer, K., 1-55, 1984.

Schmidtke, G.: Solar EUV irradiance from the San Marco ASSI: A reference spectrum, Geophys. Res. Lett., 19, 2175-2178, 1992.

Schweizer, W. and Schmidtke, G.: High-resolution extremeultraviolet solar spectrum recorded with a diffraction-filter spectrograph, The Astrophys. J., 169, L27-L29, 1971.
Schmidtke, G., Eparvier, F. G., Solomon, S. C., Tobiska, W. K., and Woods, T. N.: The TIGER (thermospheric-ionospheric geospheric research) program: Introduction, Adv. Space Res., 37, 194-198, 2006.

Schmidtke, G., Knothe, M., and Heidinger, F.: Magnetic electron multipliers: efficiency changes, Appl. Opt., 14, 1645-1648, 1975.

Schmidtke, G., Nikutowski, B., Jacobi, Ch., Brunner, R., Erhardt, Ch., Knecht, S., Scherle, J., and Schlagenhauf, J.: Solar EUV Irradiance Measurements by the Auto-Calibrating EUV Spectrometers (SolACES) aboard the International Space Station (ISS), Solar Physics, 288/2, doi:10.1007/s11207-013-0430-5, 2014.

Schmidtke, G., Schweizer, W., and Knothe, M.: The AEROS-EUV Spectrometer, J. Geophys., 40, 577-584, 1974.

Schmidtke, G., Seidl, P., and Wita, C.: Airglow-solar spectrometer instrument (20-700 nm) aboard the San Marco D satellite, Appl. Opt., 24, 3206-3213, 1985.

Solomon, S. C., Bailey, S. M., and Woods, T. N.: Effects of solar X-rays on the lower ionosphere, Geophys. Res. Lett., 28, 21492152, 2000.

Unglaub, C., Jacobi, Ch., Schmidtke, G., Nikutowski, B., and Brunner, R.: EUV-TEC proxy to describe ionospheric variability using satellite-borne solar EUV measurements: First results, Adv. Space Res., 47, 1578-1584, 2011.

Woods, T. N., Francis, G. E., Bailey, S. M., Chamberlin, P. C., Lean, J., Rottmann, G. J., Solomon, S. C., Tobiska, W. K., and Woodraska, D. L.: Solar EUV Experiment (SEE): Mission overview and first results, J. Geophys. Res., 110, A01312, doi:10.1029/2004JA010765, 2005.

Woods, T. N., Eparvier, F. G., Hock, R., Jones, A. R., Woodraska, D., Judge, D., Didkovsky, L., Lean, J., Mariska, J., Warren, H., McMullin, D., Chamberlin, P., Berthiaume, G., Bailey, S., FullerRowell, T., Sojka, J., Tobiska, W. K., and Viereck, R.: Extreme ultraviolet Variability Experiment (EVE) on the Solar Dynamics Observatory (SDO): Overview of science objectives, instrument design, data products, and model developments, Solar Phys., 275, 115-143, 2012. 